\title{
Beta-bends as an example of conformationally predetermined segments of Protein. Conditions of stabilization of the structure and role of context
}

\author{
Anastasia A. Anashkina \\ EIMB RAS, Moscow, Russia \\ nastya@eimb.ru \\ Vladimir O. Chekhov \\ EIMB RAS, Moscow, Russia \\ bock@eimb.ru
}

\author{
Ivan Yu. Torshin \\ FIC IU RAS, Moscow, Russia \\ tiy135@yahoo.com \\ Leonid A. Uroshlev \\ IGG RAS, Moscow, Russia \\ leoniduroshlev@gmail.com
}

\author{
Natalia G. Esipova \\ EIMB RAS, Moscow, Russia \\ nge@eimb.ru \\ Vladimir G. Tumanyan \\ EIMB RAS, Moscow, Russia \\ tuman@eimb.ru
}

\begin{abstract}
Conformations of the local protein structures is determined by the balance of their own energy characteristics and the context (flanking regions). The conformation of a given fragment becomes predetermined if the contribution of the context is clearly defined. Beta bends were chosen as the object of the study due to availability of formalised classification of this structure, small number of variables that determine the conformation of a segment, as well as the beta bends high prevalence in the three-dimensional structures of proteins. Using the PDB data, a complete cluster analysis of the conformationally predetermined beta-bend segments of the four main types was performed. Statistical data on the conformational parameters, amino acid composition and the amino acid sequences in beta-bends and their flanking regions were summarized. Factors have been established that stabilize the beta bend (additional hydrogen bonds between the residues involved in the bend) and the beta hairpin, flanking the bend (presence of a conformationally stable peptide). The existence of such stabilizing hydrogen bonds is confirmed by quantum chemical calculations of the energy of the fragment including bending. Stabilization of the hairpin neck is achieved by a conformationally stable hexapeptide in the conformation of the beta strand that is included in the structure, which is also confirmed by the method of denaturing molecular dynamics.
\end{abstract}

Keywords - beta-bends, conformational analysis, predetermined conformations, quantum chemistry, molecular dynamics

Motivation and aim

Motivation

The solution of the problem of protein structure can be reached it seems, by pursuing the studies of multi-scale components of the native protein structure. And though the purely structural aspects of these units are described sufficiently well and exhaustively, the issues of their stabilization and conditions for the formation of local structures are only waiting to be resolved. To approach these issues we employ the example of beta bends, the local structures whose conformations are characterized by a small number of the degrees of freedom.

Aim

Geometric aspects of the formation of beta-bends were explained by us earlier on the basis of the idea of a topological lock $[1,2]$, where closing a pseudocycle through a hydrogen bond reduces the number of significant independent parameters determining the conformation to four. Thus the number of theoretically possible structures decreases to two. Moreover, one solution corresponds to an experimentally determined structure, and the second solution cannot be realized due to the unacceptable atom-atom contacts [2]. The purpose of this study is to establish the energy aspects of the formation of predetermined conformations, including the contribution of flanking regions.

\section{Methods}

To calculate the geometry of molecules and optimize energy, we used the methods of clustering, Voronoi-Delaunay decomposition, enumeration of conformations, distance geometry, AM1, as well as the ab initio method in the approximation of $6-31++\mathrm{G} *$ with the MP2 correlation.

\section{Results}

Using the data of PDB database (including structures solved by the neutron diffraction method), a cluster analysis was performed of the four main types of beta bends identified by us as conformationally predetermined. Statistical data were collected on the conformational parameters, amino acid composition and amino acid sequences (including assessment of the residue conservation) in the beta-bends and flanking regions. A quantum chemical calculation of the change in total energy depending on the orientation in space of the NH-bond of the second residue in the beta-bend, made it possible to draw a conclusion that an additional $\mathrm{H}$ bond is formed between the carbonyl oxygen of the residue $i$ in the beta-bend and the amine nitrogen of the residue $i+2$. Another source of stabilization of the local structure as a whole is the confomationally stable (adopting the beta-strand conformation) peptide WKVEVND that secures the neck of the beta hairpin.

\section{ACKNOWLEDGMENT}

Supported by the RFBR grant 20-04-01085.

\section{REFERENCES}

[1] Uroshlev, L.A., Torshin, I.Y., Batyanovskii, A.V., Esipova, N.G. and Tumanyan, V.G., 2015. Disallowed conformations of a polypeptide chain as exemplified by the $\beta$-turn of the $\beta$-hairpin in the $\alpha$-spectrin SH3 domain. Biophysics. 60(1): 1-9.

[2] Uroshlev, L.A., Torshin, I.Y., Batyanovskii, A.V., Esipova, N.G. and Tumanyan, V.G., 2019. Predetermined Conformations in Bends of Polypeptide Chains: A Geometric Analysis. Biophysics, 64(2): 195202. 